\title{
Bilateral bipartite origin of the posterior belly of digastric muscle: a clinico-anatomical appraisal
}

\author{
Vandana Mehta, Vanita Gupta, Jyoti Arora, Yogesh Yadav, Rajesh Kumar Suri, Gayatri Rath \\ Department of Anatomy, Vardhaman Mahavir Medical College \& Safdarjung Hospital, New Delhi, India
}

\begin{abstract}
Anomalous digastric muscle may be an incidental finding during routine dissections or autopsies which may be asymptomatic or symptomatic. While performing routine educational dissection of the submandibular region of the neck, we encountered a unique bilateral composition of the digastric muscle. The posterior belly of digastric displayed a bifid origin. Two muscle bellies emanating from the digastric notch of the mastoid part of temporal bone were found to unite to form a common belly which later joined the intermediate tendon through which it was connected to the anterior belly. The innervation of this accessory muscle also displayed interesting variation. Although on the right side the innervation was solely derived from the facial nerve as is the usual case, an additional twig from the nerve to splenius capitis supplied the accessory belly on the left side. Acquaintance with the variations of this important muscle of the submandibular region is extremely important for radiologists while interpretation of MRI and CT scans and for surgeons performing reconstructive procedures. Additionally, in view of vital neurovascular structures related to the posterior belly of digastric, lack of caution on the part of surgeon may result in severe complications. The present study was designed to elucidate a rare but an important variation of the digastric muscle thereby apprising the head and neck surgeons in their operative endeavors of this region.
\end{abstract}

Key words: anatomy; anomaly; clinical importance; digastric; muscle; variation

Anatomy 2011;5:44-47, (c) 2011 TSACA

\section{Introduction}

The paired digastric muscles constitute an important part of suprahyoid muscle group. The posterior belly of digastric muscle forms a vital anatomical landmark and has several crucial neurovascular relations. It arises from the digastric notch on the medial aspect of mastoid process. The triangular fleshy belly tapers to an intermediate tendon, which is held beneath a fibrous sling attached to the junction of the body and greater horn of the hyoid bone. ${ }^{[1,2]}$ In view of the important neurovascular structures such as the XIth and XIIth cranial nerves, internal jugular vein and internal carotid artery lying deep to the posterior belly, any variation relating to it especially an accessory belly deserves special mention in anatomical literature. A form of trigastric muscle has been described where the supernumerary third head was emanating from the mandible or from the splitting of stylohyoid muscle. The superficial part of the muscle escorted the posterior belly of the digastric to gain attachment to the intermediate tendon of the digastric muscle..$^{[3]}$

Any interventional surgery or any radiological procedure in this region will require a precise knowledge of the anatomy of this region. Additionally some clinicians recommend palpation of the post belly of digastric in case of temperomandibular disorders. ${ }^{[4]}$ Numerous earlier studies about digastric muscle variations have reports pertaining to the anterior belly of the muscle, whereas the variations of posterior belly are largely unavailable. ${ }^{5}$ To the best of our knowledge, the bilateral bipartite ori- 
gin of posterior belly of digastric muscle has yet to be cited in literature. Digastric muscle stabilizes the hyoid bone in addition to assisting in jaw movement, particularly depression of mandible. ${ }^{[6]}$ Normal function of this muscle should be borne in mind while evaluating functional disorders of the oral apparatus.

\section{Case Report}

The present anomaly was detected in a 45-year-old male cadaver during the course of preclinical educational training session. The suprahyoid region was dissected and the posterior belly of digastric muscle was exposed. The present case specimen was characterized by the presence of bilateral bipartite origin of the posterior belly of digastric muscle. These supernumerary bellies emanated from the medial aspect of the mastoid notch and were seen to fuse with the main belly of the posterior part of the digastric muscle.

On the right side, each part of the posterior belly of digastric showed in superior and posterior positions. These superior and inferior bellies were measured as 4.1 $\mathrm{cm}$ and $4.3 \mathrm{~cm}$ in length, respectively. Both the bellies were fleshy and joined to form a single muscle which was $1 \mathrm{~cm}$ in length. The intermediate tendon was measured as $2.8 \mathrm{~cm}$ in length. Both the bellies were found to be innervated by the facial nerve. The stylohyoid muscle was observed to attach to the intermediate tendon of the digastrics (Figure 1).

On the left side, the positions of each part of the posterior belly of digastric showed in medial and lateral positions. These medial and lateral bellies were measured as $3.4 \mathrm{~cm}$ and $3.1 \mathrm{~cm}$, respectively (Figure 2). The common muscle belly was $1.6 \mathrm{~cm}$ long and the intermediate tendon was measured as $3 \mathrm{~cm}$ in length. The left lateral belly interestingly received innervation from the nerve to splenius capitis, in addition to the usual nerve supply from the facial nerve. The remainder of the musculature in the vicinity displayed usual morphology and innervation.

\section{Discussion}

Several studies in the past have elucidated various anomalies of the anterior belly of digastric. ${ }^{[7]}$ These variations have been reported unilaterally as well as bilaterally. ${ }^{8} \mathrm{We}$ agree with Testut's study, wherein he coined the term "trigastric muscle" for the supernumerary fascicle of anterior belly of digastric. We wish to adopt the terminology used by Testut, but the 'trigastric' encountered in our case specimen consists of a supernumerary posterior belly of digastric instead of the anterior belly. A study describing an accessory median digastric muscle has also been reported in literature..$^{[9]}$ Nevertheless, none of the above described anomalies resemble the accessory muscle encountered in the present case study. An interesting report by revealed an accessory muscle of posterior belly of digastric inserted into the lateral border of strap muscles. ${ }^{[10]}$ The muscle in the present study is unusual in view of its bipartite origin displayed bilaterally.

We strongly believe that muscular variation found in the present study is extremely unique and has not been

Figure 1. Dissection of the right digastric triangle of the neck. $A B$ : anterior belly of digastric; PB1: posterior belly (superior); PB2: posterior belly (inferior); SH: stylohyoid muscle; IT: intermediate tendon.

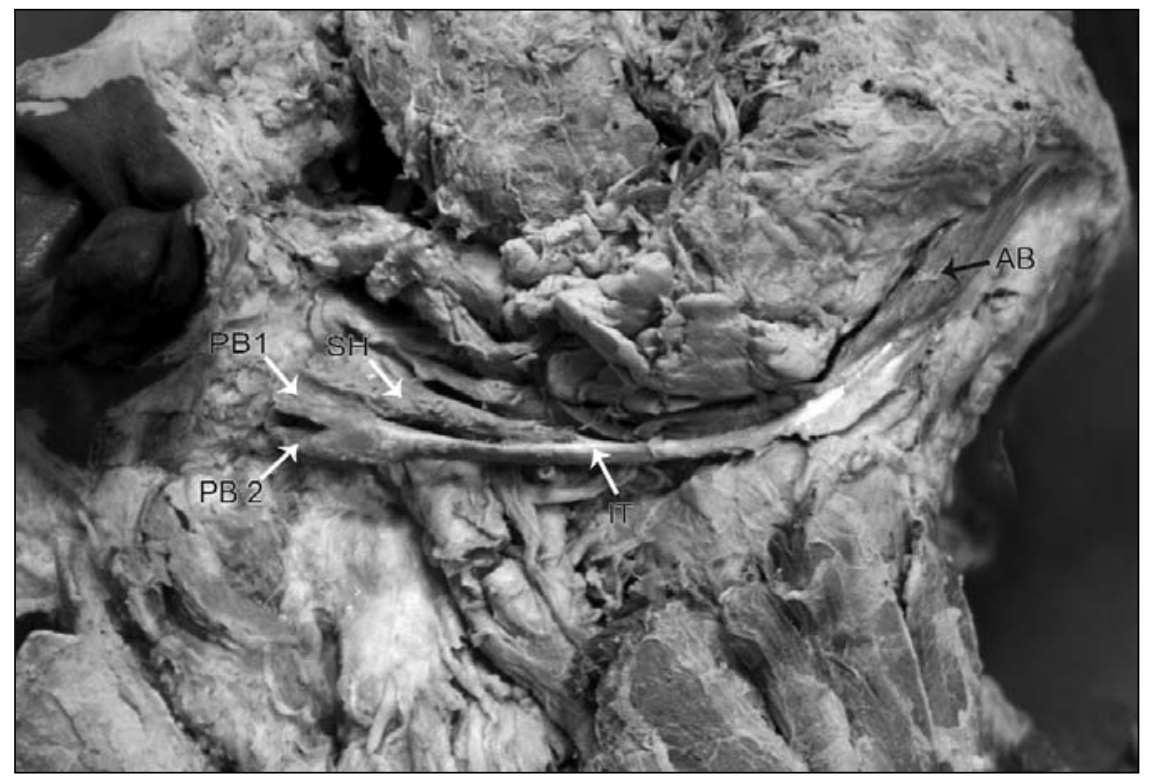




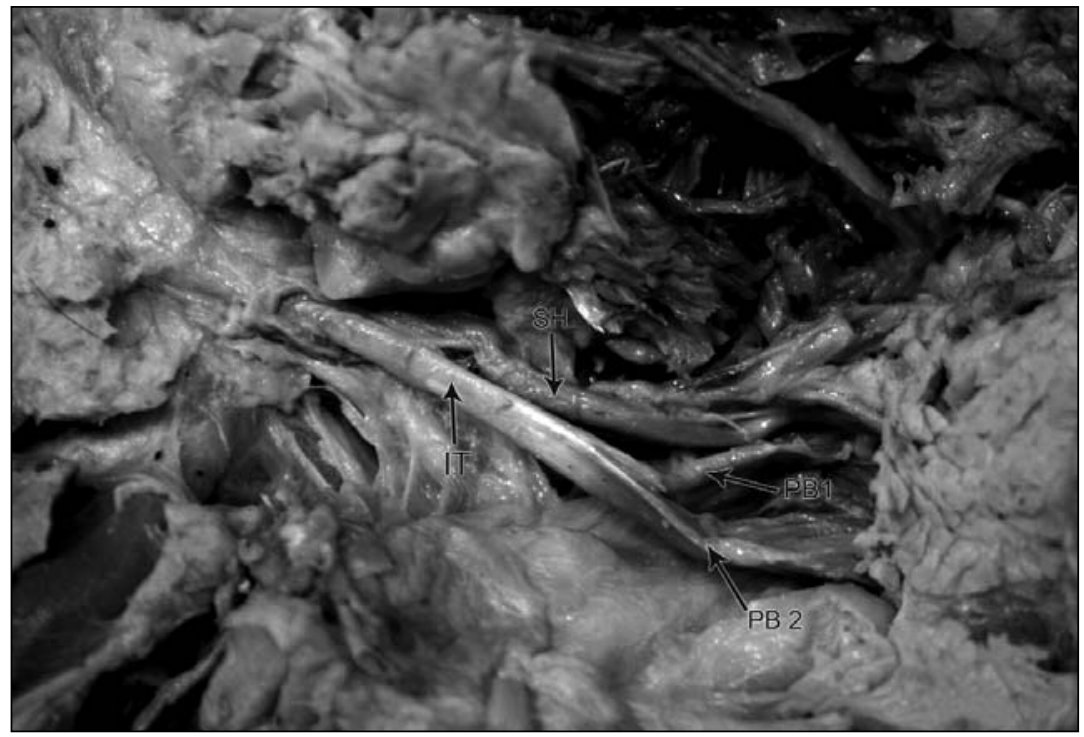

Figure 2. Dissection of the left digastric triangle of the neck. PB1: posterior belly (medial); PB2: posterior belly (lateral); SH: stylohyoid muscle; IT: intermediate tendon.

reported yet. The anterior belly of digastric is used for muscle transfers in case of iatrogenic damage to mandibular branch of facial nerve. We opine that the supernumerary heads of digastric muscle as found in the present study could also possibly be utilized for reconstructive purpose. Moreover, it seems to be a feasible option as the main bellies of digastrics would not have to be sacrificed for the procedure. Since the anterior belly of digastric and mylohyoid muscles are derivatives of the first pharyngeal arch, anomalies of these two muscles often co-exist. ${ }^{[11]}$ The posterior belly of digastric muscle is derived from the second pharyngeal arch.

It is a well established fact that cells of origin of the muscle mass migrate from the para-axial mesenchyme of the somitomeres. Another interesting fact about the development of these muscles is that surrounding neural cells contribute to this lineage. It is also recognized that the HOX genes play an important role in the organization of nervous system and in the differentiation of branchial arch structures. Additionally, the digastrics muscle may be viewed as a composite owing to its compound origin reflected by the dual innervation of the muscle. ${ }^{[12]}$ The above explanation has been validated by the law of fusion which describes that muscles are associated with their nerve at an early stage of development. The fusion of the two muscle masses at a later stage yields a muscle supplied by two different nerves.

Although the additional muscle bellies in the present case report may be asymptomatic clinically, but their presence may prove to be important in the operations involving the submandibular region. Further, the unusual muscle constitution of the present case may also pos- sibly result in compression of the vital neurovascular structures traversing deep to posterior belly of digastric. Familiarity with the muscular variations of this region in case of reconstructive surgeries of the neck is essential while carrying out placement of myocutaneous flaps. ${ }^{[13]}$

In temporomandibular disorders, palpation of the posterior belly of digastric is a mandatory procedure. An anomalous posterior belly may possibly lead to perplexing situations while diagnosing these disorders. ${ }^{[4]}$ Electromyography suggested that the paired digastrics act together and are secondary to lateral pterygoid in mandibular depression. ${ }^{[6]}$ It is our speculation that the accessory heads of the posterior belly of digastric muscle may serve to augment the mandibular depression. Additionally, radical suprahyoid neck procedures are often required while removing metastatic lymph nodes in carcinoma involving floor mouth. ${ }^{[14]}$ In such dissections, posterior belly of digastric muscle serves as a useful landmark. Unexpected presence of an additional belly may be mistaken either for a pathological tumor or any other soft tissue mass, and may be sacrificed by mistake.

The accidental removal of this additional head of posterior belly of digastric would result in incomplete removal of pathological tissues resulting in recurrence in case of malignancy.

Furthermore, CT and MRI scans are valuable investigative procedures in assessment, prognosis and treatment planning of tumors of head and neck region. Awareness of muscular variations in this region could aid in expelling any doubt about pathological lesions. It is well documented that neoplasms especially after radio- 
therapy develop reduced intensity, mimicking the signals obtained from the muscles on MRI. Thus, an unexpected occurrence of additional posterior belly of digastric may lead to mistaken diagnosis and maybe mis-interpreted as a healing lesion. An accessory digastric muscle owing to the location and tissue density may be erroneously deciphered as a normal or metastatic lymph node or pseudo masses on CT scan examination. Rotation or any other movement of the head may lead to an erroneously enlarged size of the normal digastric during imaging of the submental region. ${ }^{[15,16]}$ Admittedly, clinical history of the individual was not obtainable in the present case. Therefore, it is rather hard to comment on the correlation between morphological findings and clinical symptomatology of the individual.

Nevertheless, as anatomists it is our responsibility to document these variations so as to caution and reinforce awareness among surgeons and radiologists while dealing with this vital region. The additional twig derived from the nerve to splenius capitis and supplying the posterior belly of digastric of the left side, is an interesting observation. It logically implies that the accessory head of the left posterior belly of digastric might be the result of dorsoventral migration of few myotomes from the suboccipital region. Further, the additional innervation of the accessory head of posterior belly of digastric muscle should possibly augment its suitability for its use in muscle transfer operations.

Our premise is that precise knowledge of the muscular variations of this vital region is of paramount relevance in the interpretation of radiological procedures and surgical explorative procedures.

\section{Conclusion}

Although variations in the muscular anatomy of the posterior belly of digastric are rarely reported in anatomical archives, these are nevertheless important while performing radiological procedures. These observations assume special significance while exploring the submental and submandibular regions for tumors and metastases as well as for other operative maneuvers.

\section{References}

1. Sinnatamby CS. Head and neck and spine. In: Last's Anatomy. 10th ed. Edinburgh: Churchill Livingstone; 1998. p. 327.

2. Brand RW, Iselhard DE. Anatomy of Orofacial Structures. 7th ed. St Louis: Mosby; 1998.p.341.

3. Bergmann RA, Afifi AK, Miyauchi R. Abductor digiti minimi pedis. Illustrated encylopedia of human anatomic variation: Opus I: Muscular System: Alphabetical listing of muscles: D. http://www.anatomyatlases.org/AnatomicVariants/MuscularSyste $\mathrm{m} /$ Text/D/06Digastricus.shtml (30th of September, 2010).

4. Turp JC, Arima T, Minagi S. Is posterior belly of digastric palpable? Qualitative and systematic review of literature. Clin Anat 2005;18:318-22.

5. Sargon MF, Çelik HH. An abnormal digastric muscle with three bellies. Surg Radiol Anat 1994;16:215-6.

6. Moyers RE. Electromyographic analysis of certain muscles involved in tempro-mandibular movements. Am J Orthodont 1950;36:481-515.

7. Michna H. Anatomical anomalies of human digastric muscle. Acta Anat 1989;134:263-4.

8. Testut L, Latarjet A. Traite d' Anatomie Humaine. 9th ed. Paris: Gaston Doin \& Cie.: 1948.p.68-70.

9. Guelfguat M, Nurbhai N, Solounias N. Median accessory digastric muscles: radiological and surgical correlation. Clin Anat 2001;14: 42-6.

10. Özgürsoy OB, Küçük B. Unique variation of digastric muscle: a confusing landmark for head \& neck surgeons. Acta Otolaryngol 2006;126:881-3.

11. Ziolkouski M, Marek J, Klak A. The human digastric muscle in fetal period. Folia Morphol 1984;3:243-9.

12. Shinohara H. A warning against revival of classical tenets of gross anatomy related to nerve muscle specificity. J Anat 1996;188:2478.

13. Conley J, Patow C. Flaps in Head and Neck Surgery. 2nd ed. New York: Thieme; 1989.p.215-58.

14. Suen JY, Stern SJ. Cancer of neck. In: Myers EN, Suen JY, eds. Cancer of Head and Neck. 3rd ed. Philadelphia: WB Saunders Co.; 1996.p.462-84.

15. Muraki AS, Mancuso AA, Harnsberger HR, Johnson LP, Meads GB. CT of oropharynx, tongue base and floor of mouth. Normal anatomy and range of variation and application in staging cancer. Radiology 1983;148:725-31.

16. Sevinç Ö, Çetin ZA, Barut C, Büken B, Arifoğlu Y. A complex variation of the digastric muscle: a case report. Anatomy 2009;3:72-4.

Correspondence to: Vandana Mehta, MS

Department of Anatomy

VMMC \& SJH, New Delhi, India

e-mail: drvandanamehta@gmail.com

Conflict of interest statement: No conflicts declared. 